\title{
An estimate of the D/H ratio in Jupiter and Saturn's regular icy satellites - Implications for the Titan Huygens mission
}

\begin{abstract}
O. Mousis
CNRS-UMR 6091, Observatoire de Besançon, BP 1615, 25010 Besançon Cedex, France

Received 20 October 2003 / Accepted 18 November 2003

Abstract. The solar nebula model described by Dubrulle (1993) and Drouart et al. (1999) is used to predict the $\mathrm{D} / \mathrm{H}$ ratio in $\mathrm{H}_{2} \mathrm{O}$ ice of both Jovian and Saturnian regular satellite systems. These calculations take into account recent scenarios of formation of Jupiter and Saturn's large regular icy satellites developed from evolutionary turbulent models of the surrounding subnebulae. These scenarios argue that the Saturnian and Jovian subdisks were cold enough to prevent the vaporization of volatile species trapped in solids which produced the regular icy satellites. The hydrated solids formed in the feeding zones of Jupiter and Saturn during the cooling of the solar nebula. Hence, the $\mathrm{D} / \mathrm{H}$ ratio in $\mathrm{H}_{2} \mathrm{O}$ ice of hydrated solids is likely the result of the isotopic exchange between HD and HDO which occurred in the solar nebula prior to the condensation of water. In these conditions, the $\mathrm{D} / \mathrm{H}$ ratio in the regular icy satellites of Jupiter and Saturn is estimated to be between 3.8 and 4.7, and between 4.8 and 6.8 times the protosolar value, respectively. Such estimates, compared with subsequent in situ measurements of the $\mathrm{D} / \mathrm{H}$ ratio in $\mathrm{H}_{2} \mathrm{O}$ ice of the Jovian and Saturnian regular satellite systems, should bring new constraints on the proposed scenarios of formation. This is the case with the Huygens probe mission which has the capacity of measuring the $\mathrm{D} / \mathrm{H}$ ratio in the water ice which may exist on the surface of Titan.
\end{abstract}

Key words. planets and satellites: formation - solar system: formation

\section{Introduction}

The formation of major regular icy satellites surrounding Saturn and Jupiter has been revisited recently by several authors (Mousis et al. 2002a; Canup \& Ward 2002; Mosqueira \& Estrada 2003a,b; Mousis \& Gautier 2004). Both Canup \& Ward (2002) and Mosqueira \& Estrada (2003a,b) proposed stationary models of the Jovian and Saturnian subnebulae in order to describe the conditions of accretion of the regular satellites. However, the fact that these authors did not employ time-dependent models prevented any investigation of the temporal evolutions of the carbon, nitrogen and isotopic gas phase chemistries in the Jovian and Saturnian subdisks. As a result, no predictions of observational tests have been qualitatively described by the authors in the icy regular satellites to constrain their scenarios of formation. On the other hand, Mousis et al. (2002a) and Mousis \& Gautier (2004) (hereafter labelled Papers I and II) developed evolutionary turbulent models of the Saturnian and Jovian subnebulae which were derived from the solar nebula analytical model of Dubrulle (1993). They explored the thermodynamical conditions existing in the Saturnian and Jovian subnebulae in order to follow the chemical evolution of carbon and nitrogen species in these environments. Paper I proposed a scenario of the formation of Titan

\footnotetext{
* e-mail: 0livier.Mousis@obs-besancon.fr
}

compatible with its current atmospheric molecular composition. Based on this scenario, Mousis et al. (2002b) provided an interpretation of the $\mathrm{CH}_{3} \mathrm{D} / \mathrm{CH}_{4}$ ratio measured in the atmosphere of Titan. With a similar approach, Paper II estimated the per mass abundances of $\mathrm{NH}_{3}, \mathrm{~N}_{2}, \mathrm{CO}$ and $\mathrm{CH}_{4}$ with respect to $\mathrm{H}_{2} \mathrm{O}$ in the interiors of Ganymede and Callisto, thus providing an explanation for the suspected presence of ammonia in the subsurface oceans of these satellites.

In Papers I and II, the authors advocated that the solids which formed Titan, as well as Ganymede and Callisto, were produced in the feeding zones of Saturn and Jupiter prior to the formation of their surrounding subnebulae. They argued that carbon and nitrogen volatiles were trapped in the form of hydrates and clathrate hydrates in the feeding zones of both giant planets before being incorporated in planetesimals embedded in the cold outer zones of their corresponding subnebulae. In these conditions, Titan, as well as Ganymede and Callisto, is assumed to have formed from these icy planetesimals which migrated inward from the outer zones of the Saturnian and Jovian subdisks. Some observational tests have been provided by Papers I and II to check the validity of their scenarios of formation. Paper I predicted the ratios of some noble gas abundances to that of carbon in the atmosphere of Titan today which may be measured by the Huygens probe. However, up until now, these volatile species have not been detected in the 
atmosphere of Titan. Moreover, the existence of important amounts of $\mathrm{NH}_{3}, \mathrm{~N}_{2}, \mathrm{CO}$ and $\mathrm{CH}_{4}$ predicted by Paper II on the surfaces of Ganymede and Callisto is subject to caution. Indeed, the alteration of the surface composition of these icy satellites by large impactors and space weathering during the lifetime of the solar system is likely to impede the subsequent detection of carbon and nitrogen species, due to their high volatility. Hence, some additional observational tests have to be formulated to assess the proposed scenarios of formation.

In this work, we focus on the possibility of determining the value of the $\mathrm{D} / \mathrm{H}$ ratio in $\mathrm{H}_{2} \mathrm{O}$ ice taking part in the composition of the regular icy satellites of both Jovian and Saturnian systems. Since water ice is expected to exist on the surface of all the icy satellites of these systems, including Titan (Griffith et al. 2003), we believe that the proposed observational test is more constraining than the previous ones described by Papers I and II. The present work is based on the studies of Drouart et al. (1999) and Mousis et al. (2000) who provided a description of the evolution of the $\mathrm{D} / \mathrm{H}$ ratio in $\mathrm{H}_{2} \mathrm{O}$ in the solar nebula. In Sect. 2, we use the range of plausible solar nebula models they have determined to calculate the range of $\mathrm{D} / \mathrm{H}$ enrichments $f$ in water, with respect to the solar value in hydrogen, in both Jupiter and Saturn's feeding zones. From the calculated values of $f$ and from the scenarios of formation derived from Papers I and II, we then determine in Sect. 3 the existing $\mathrm{D} / \mathrm{H}$ ratio in $\mathrm{H}_{2} \mathrm{O}$ ice of the Jovian and Saturnian regular icy satellites. In Sect. 4 , we discuss the implications of a possible measurement by the Huygens probe of the $\mathrm{D} / \mathrm{H}$ ratio in $\mathrm{H}_{2} \mathrm{O}$ ice on the surface of Titan.

\section{Evolution of the $\mathrm{D} / \mathrm{H}$ ratio in $\mathrm{H}_{2} \mathrm{O}$ in the solar nebula}

We follow the works of Drouart et al. (1999) and Mousis et al. (2000) who described the evolution of the $\mathrm{D} / \mathrm{H}$ ratio in $\mathrm{H}_{2} \mathrm{O}$ in the solar nebula. Drouart et al. (1999) physically interpreted the measurements of the $\mathrm{D} / \mathrm{H}$ ratio in LL3 meteorites, and in comets P/Halley, C/1996 B2 (Hyakutake), and C/1995 O1 (Hale-Bopp). These authors employed the time dependent turbulent model developed for the solar nebula by Dubrulle (1993) which depends on three physical parameters: the initial mass of the nebula $M_{\mathrm{D} 0}$, its initial radius $R_{\mathrm{D} 0}$, and the coefficient of turbulent viscosity $\alpha$, derived from the prescription of Shakura $\&$ Sunyaev (1973). They calculated the deuterium enrichment factor $f$ in water, with respect to the protosolar value in hydrogen, by integrating the equation of diffusion in the solar nebula, as a function of the heliocentric distance and time. The comparison of the obtained value of $f$ to observations allowed Drouart et al. (1999) to determine a range of possible values for $M_{\mathrm{D} 0}, R_{\mathrm{D} 0}$, and $\alpha$. Better constraints on these parameters were obtained by Mousis et al. (2000) who reunified the $\mathrm{D} / \mathrm{H}$ ratio in proto-Uranian and proto-Neptunian ices with the cometary value by taking into account the recent interior models of Uranus and Neptune calculated by Podolak et al. (2000). Thus, the coefficient of turbulent viscosity $\alpha$ was estimated to be of the order of 0.003 .
For the needs of the present work, we report in Table 1 the physical parameters of the minimum mass and maximum mass models of the solar nebula constrained by Mousis et al. (2000). We have integrated the equation of diffusion for these two extreme mass models to follow the temporal evolution of the $\mathrm{D} / \mathrm{H}$ ratio in $\mathrm{H}_{2} \mathrm{O}$ in the solar nebula (see Drouart et al. 1999 for details), and more specifically in the feeding zones of proto-Jupiter and proto-Saturn. We follow the strategy described by Drouart et al. (1999) for setting the initial value of $f$ at $t=0$. We have assumed $f(R)=31$ at $t=0$ for $\mathrm{D} / \mathrm{H}$ in water. This value corresponds to that measured in the highly enriched component $\left(\mathrm{D} / \mathrm{H}=(73 \pm 12) \times 10^{-5}\right)$ found in LL3 meteorites (Deloule et al. 1998) which is compared to a protosolar value assumed to be equal to $(2.35 \pm 0.3) \times 10^{-5}$ (Mousis et al. 2002b). The isotopic exchange between $\mathrm{HDO}$ and $\mathrm{H}_{2}$ occurs as long as $\mathrm{H}_{2} \mathrm{O}$ remains in the vapor phase. This implies that the value of the enrichment in microscopic ices is the one fixed at the time and at the location of the condensation of water vapor. As soon as these icy grains reached the centimeter size, they decoupled from the gas, continued their growth to form planetesimals, and orbited around the sun (Weidenschilling 1997). In this paper, we consider the case where planetesimals produced in the feeding zone of Jupiter and Saturn agglomerated only from icy grains formed locally. Another extreme case, which is not investigated here, would be to consider planetesimals formed from a mixing of grains coming from all over the nebula. This would lead to a higher D-enrichment in the icy planetesimals.

Table 1. Initial radius $R_{\mathrm{D} 0}$ and mass $M_{\mathrm{D} 0}$ of the minimum mass and maximum mass solar nebula models characterized by $\alpha=0.003$ and constrained by Mousis et al. (2000).

\begin{tabular}{lcc}
\hline \hline & $R_{\mathrm{D} 0}(\mathrm{AU})$ & $M_{\mathrm{D} 0}\left(M_{\odot}\right)$ \\
\hline Minimum mass solar nebula & 15 & 0.06 \\
Maximum mass solar nebula & 27 & 0.3 \\
\hline
\end{tabular}

Figure 1 represents the value of the D-enrichment $f$ in $\mathrm{H}_{2} \mathrm{O}$ in icy planetesimals as a function of their distance to the sun in $\mathrm{AU}$ in the cases of the minimum mass and maximum mass solar nebula models. Assuming that Jupiter and Saturn were formed at their current locations in the solar system (Pollack et al. 1996), the plausible range of values of $f$ in the solids produced in the feeding zones of both giant planets is given by the vertical bold solid lines in Fig. 1. This gives a D-enrichment $f$ in $\mathrm{H}_{2} \mathrm{O}$ in icy planetesimals which is between 3.8 and 4.7, and between 4.8 and 6.8 times the protosolar value in the feeding zones of Jupiter and Saturn, respectively.

Following the scenario of Pollack et al. (1996), Jupiter and Saturn were formed in three phases from gas and solids present in their feeding zones during the cooling of the solar nebula. Coradini et al. (1995) calculated that Jupiter and Saturn's circumplanetary disks were generated by the hydrodynamical collapse of the gas onto the core of both giant planets which occured during the last phase of their formation. This implies that the material (gas + solids) which initially composes the 
circumplanetary disks originates from the feeding zones of Jupiter and Saturn. Hence, the thermodynamical conditions existing in both Jovian and Saturnian subnebulae have to be examined. This will allow us to follow the subsequent evolution of the $\mathrm{D} / \mathrm{H}$ ratio in $\mathrm{H}_{2} \mathrm{O}$ contained in the icy solids which were initially included in these media. In the next section, we report the physical characteristics of Jupiter and Saturn's circumplanetary disks which are described in Papers I and II. We then discuss the implications for the resulting $\mathrm{D} / \mathrm{H}$ ratio in the $\mathrm{H}_{2} \mathrm{O}$ ice included in the composition of the regular icy satellites.

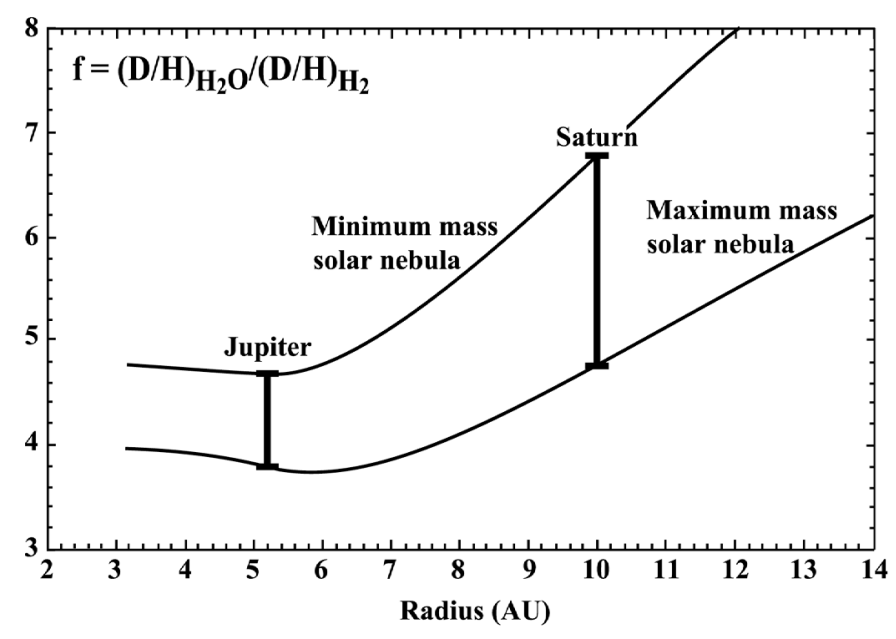

Fig. 1. Deuterium enrichment factor $f$ calculated at the epochs of the condensation of water for the minimum mass and maximum mass solar nebula models derived from Mousis et al. (2000). The vertical bold solid lines correspond to the range of values of $f$ inferred in the icy solids produced in Jupiter and Saturn's feeding zones.

\section{Formation of Jovian and Saturnian regular satellite systems from D-enriched icy planetesimals}

Figure 2 represents the temporal evolution of the mass distribution in the Jovian subnebula model described in Paper II. It can be seen that most of the mass of the subnebula (gas + gascoupled solids) remains in the outer part of the disk, where $T$ and $P$ conditions are too low to vaporize water ice. The same remark can be expressed for the model of Saturn's subnebula described in Paper I. In such conditions, one can deduce that most of the icy planetesimals initially produced in the feeding zones of Jupiter and Saturn did not outgas when they took part in the composition of the respective subnebulae. Papers I and II argued that Titan, as well as Ganymede and Callisto, were accreted at times when their regions of formation were cold enough to preserve icy planetesimals from vaporization. This argument also applies to the formation of each icy satellite produced in the Jovian and Saturnian circumplanetary disks. The zone of formation of the regular satellites was then fed by planetesimals that migrated inwards from the outer part of the subnebula.

According to the presented scenario of formation, one can deduce that the regular icy satellites of Jupiter present the same
$\mathrm{D} / \mathrm{H}$ ratio in $\mathrm{H}_{2} \mathrm{O}$ ice as the one determined in its feeding zone. Indeed, if water was preserved from vaporization in planetesimals that produced the Jovian icy satellites, no isotopic exchange occurred in the vapor phase between the trapped HDO and $\mathrm{H}_{2}$. The same condition applies to the regular icy satellites of Saturn. Therefore, the D-enrichment $f$ in the Jovian and Saturnian regular icy satellites is predicted to be between 3.8 and 4.7, and 4.8 and 6.8 times the protosolar value, respectively. With a $(\mathrm{D} / \mathrm{H})$ proto equal to $(2.35 \pm 0.3) \times 10^{-5}$ (Mousis et al. 2002b), the corresponding predicted value of the $\mathrm{D} / \mathrm{H}$ ratio in $\mathrm{H}_{2} \mathrm{O}$ in the Jovian and Saturnian regular icy satellites is between $7.8 \times 10^{-5}$ and $12.4 \times 10^{-5}$, and between $9.8 \times 10^{-5}$ and $18 \times 10^{-5}$, respectively. Subsequent in situ measurements of the $\mathrm{D} / \mathrm{H}$ ratio in $\mathrm{H}_{2} \mathrm{O}$ ice on the surfaces of the Jovian and Saturnian regular satellites, compared with the predicted values, should allow us to check the validity of the scenarios of formation proposed by Papers I and II. In the next section, we describe the prospects of the Cassini-Huygens mission concerning the measurement of the $\mathrm{D} / \mathrm{H}$ ratio in the $\mathrm{H}_{2} \mathrm{O}$ ice potentially existing on the surface of Titan and the implications for the origin of its atmospheric methane.

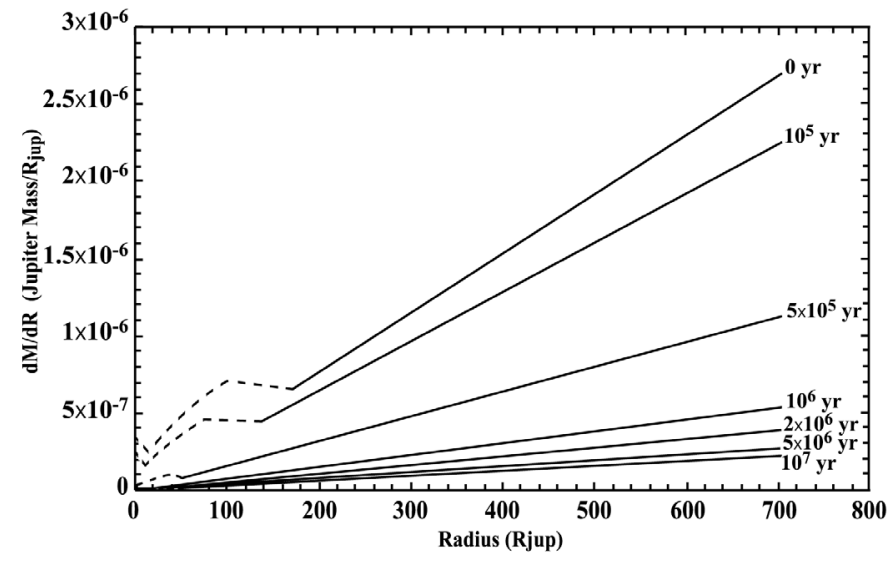

Fig. 2. Mass distribution in the Jovian subnebula described by Paper II, for various values of $t$ in years. The Jovian subnebula is characterized by the parameters $M_{\mathrm{D}}=0.001 M_{\text {Jup }}, R_{\mathrm{D}}=704 R_{\text {Jup }}$, and $\alpha=0.0004$. The time $t=0$ corresponds to the epoch when the formation of Jupiter was completed. Dashed curves represent the zone of the subnebula where $T$ and $P$ are high enough to vaporize $\mathrm{H}_{2} \mathrm{O}$ ice. Plain curves correspond to the zone of the subnebula where $T$ and $P$ conditions are low enough to preserve $\mathrm{H}_{2} \mathrm{O}$ ice from evaporation. With time, the cold zone expands inward.

\section{Implications for the Titan Huygens mission}

Griffith et al. (2003) derived a spectrum of Titan's surface visible with narrow windows centered at $0.83,0.94,1.07,1.28$, 2.0, 2.9, and 5.0 micrometers and detected features characteristic of water ice. Hence, despite the hundreds of meters of organic liquids and solids hypothesized to exist on Titan's surface, its icy bedrock lies extensively exposed. The detection of such features provides additional prospects for the CassiniHuygens mission whose arrival in the Saturn system is expected in the middle of 2004. In the case of a successful landing of 
the Huygens Titan probe on the icy surface of the satellite in January 2005, the gas chromatograph and mass spectrometer (GCMS) instrument has the ability to measure the $\mathrm{D} / \mathrm{H}$ ratio in $\mathrm{H}_{2} \mathrm{O}$ ice (Raulin 2003, private communication). Such a measurement, compared with the value predicted in the present work, should bring further constraints to the scenario of the formation of Titan proposed by Paper I.

The value of the $\mathrm{D} / \mathrm{H}$ ratio measured in the atmospheric methane of Titan $\left(8.7\left(\begin{array}{c}+3.2 \\ -1.9\end{array}\right) \times 10^{-5}\right.$, Coustenis et al. 2003) is close to the predicted value of the $\mathrm{D} / \mathrm{H}$ ratio in $\mathrm{H}_{2} \mathrm{O}$ ice. Up until now, two physical processes have been proposed to explain the D-enrichment in the methane of Titan. The first process, described by Pinto et al. (1986) and Lunine et al. (1999), suggests that the photodissociated methane is replenished by evaporation from a reservoir located on the surface. Lunine et al. (1999) calculated that the solar photolysis of the initially nonD-enriched $\mathrm{CH}_{4}$ over a 4.5 byr timescale can explain today's observed $\mathrm{D} / \mathrm{H}$ ratio in methane, assuming a closed system for the surface-atmospheric methane system. The second process, proposed by Mousis et al. (2002b), is based on the scenario of the formation of Titan described by Paper I and reported in Sect. 3. These authors demonstrated that the observed Denrichment in methane could result from the isotopic exchange which occured between $\mathrm{CH}_{3} \mathrm{D}$ and $\mathrm{H}_{2}$ in the vapor phase in the solar nebula. This isotopic exchange stopped when methane was trapped in icy planetesimals in the form of clathrate hydrates. The physical process invoked by these authors to explain the $\mathrm{D} / \mathrm{H}$ enrichment in $\mathrm{CH}_{4}$ is then similar to the one described in this work to predict the $\mathrm{D} / \mathrm{H}$ ratio in the $\mathrm{H}_{2} \mathrm{O}$ ice. Icy planetesimals which subsequently formed Titan in the circumplanetary disk were produced in the feeding zone of Saturn and were expected to preserve their initial volatiles content. In the framework of this scenario, the authors invoked the cryovolcanism hypothesis to explain the observed D-enrichment in the atmospheric methane.

The measurement of the $\mathrm{D} / \mathrm{H}$ ratio in $\mathrm{H}_{2} \mathrm{O}$ by the GCMS instrument aboard the Titan Huygens probe is likely to confirm one of the two proposed physical processes. Indeed, if the $\mathrm{H}_{2} \mathrm{O}$ ice presents a D-enrichment close to that in methane, compared to $(\mathrm{D} / \mathrm{H})$ proto, one can expect that both $\mathrm{D} / \mathrm{H}$ ratios in $\mathrm{H}_{2} \mathrm{O}$ and $\mathrm{CH}_{4}$ were the result of an isotopic exchange in vapor phase in the solar nebula. Such a measurement would favor the scenario of the formation of Titan proposed by Paper I and the hypothesis of cryovolcanism for the origin of its atmospheric methane invoked by Mousis et al. (2002b). On the other hand, the measurement of a $\mathrm{D} / \mathrm{H}$ ratio in $\mathrm{H}_{2} \mathrm{O}$ close to $(\mathrm{D} / \mathrm{H})_{\text {proto }}$ would indicate that the observed D-enrichment in the atmospheric methane of Titan is not primordial. This would be the result of atmospheric processes like those described by Pinto et al. (1986) and Lunine et al. (1999).

Acknowledgements. Many thanks are due to Francois Raulin for his remarks on the capabilities of the GCMS instrument aboard the Huygens Titan probe. I wish to acknowledge Doug Marshall, Guy Moreels and Jean-Marc Petit for their valuable comments on the manuscript.

\section{References}

Canup, R. M., \& Ward, W. R., 2002, AJ, 124, 3404

Coradini, A., Federico, C., Forni, O., et al. 1995, Surv. Geophys., 16, 533

Coustenis, A., Salama, A., Schulz, B., et al. 2003, Icarus, 161, 383

Deloule, E., Doukhan, J.-C., \& Robert F., 1998, Geochim. Cosmochim. Acta, 62, 3367

Drouart, A., Dubrulle, B., Gautier, D., et al. 1999, Icarus, 140, 129

Dubrulle, B., 1993, Icarus, 106, 59

Griffith, C. A., Owen, T., Geballe, T. R., et al. 2003, 300, 628

Lunine, J. I., Yung, Y. K., \& Lorentz, R. D. 1999, Planet. Space Sci., 47,1291

Mosqueira, I., \& Estrada, P. R., 2003a, Icarus, 163, 198

Mosqueira, I., \& Estrada, P. R., 2003b, Icarus, 163, 232

Mousis, O., Gautier, D., Bockelée-Morvan, D., et al. 2000, Icarus, 148, 513

Mousis, O., Gautier, D., \& Bockelée-Morvan, D., 2002a, Icarus, 156, 162 (Paper I)

Mousis, O., Gautier, D., \& Coustenis, A., 2002b, Icarus, 159, 156

Mousis, O., \& Gautier, D., 2004, Planet. Space Sci., in press (Paper II)

Pinto, J. P., Lunine, J. I., Kim, S.-J., et al. 1986, Nature, 319, 388

Podolak, M., Podolak, J. I., \& Marley, M. S. 2000, Planet. Space Sci., 48, 143

Pollack, J. B., Hubickyj, O., Bodenheimer, P., et al. 1996, Icarus, 124, 62

Shakura, N. I., \& Sunyaev, R. A., 1973, A\&A, 24, 337

Weidenschilling, S. 1997, Icarus, 127, 290 